\title{
Primary Ependymoma of the Ovary Mimicking as Yolk Sac Tumor: A Rare Case Report
}

\begin{abstract}
Ependymoma is a glioma with the differentiation toward ependymal cells that usually arise in the central nervous system. Primary ovarian ependymoma is a rare gynecologic malignancy, of which very few cases have been published. Here, we present the case of a 21-year-old woman who presented with a pelvic mass. Ultrasonography revealed a solid cystic mass in the left adnexa. The patient underwent left oophorectomy followed by cytoreductive completion surgery. On histopathological examination, a possibility of primary glial and neuronal tumor of the ovary was made. The final diagnosis of primary ependymoma of the ovary was arrived upon due to strong positivity for glial fibrillary acidic protein and S100, along with positivity for pancytokeratin, epithelial membrane antigen, vimentin, estrogen receptor, and progesterone receptor on immunohistochemistry. Although a rare diagnosis, when presented with an ovarian mass in a young patient, this diagnosis must be borne in mind.
\end{abstract}

Keywords: Ependymoma, glial fibrillary acidic protein, ovary

\section{Introduction}

Ependymomas usually arise from the wall of the ventricles or the central canal of the spinal cord. Extracranial and extraspinal ependymomas are extremely rare but have been reported in the ovary, broad ligament, sacrococcygeal region, lung, and mediastinum. ${ }^{[1-3]}$ Compared to their central nervous system (CNS) counterparts, they show highly variable histology, often causing diagnostic errors. Here, we report the case of a young female with primary ovarian ependymoma, treated with unilateral oophorectomy followed by cytoreductive surgery with subsequent hormonal therapy.

\section{Case Report}

A 21-year-old female presented with chief complaints of lower abdominal pain for the past 1 month. Her last menstrual period was 40 days before her presentation. She had the obstetric history of a full-term normal delivery a year ago. Ultrasound of the abdomen showed a solid cystic lesion in the left adnexa and left pelvis. The solid component measured $7 \mathrm{~cm} \times 6 \mathrm{~cm}$ and the cystic component measured $8 \mathrm{~cm} \times 7 \mathrm{~cm}$.

\footnotetext{
This is an open access journal, and articles are distributed under the terms of the Creative Commons Attribution-NonCommercial-ShareAlike 4.0 License, which allows others to remix, tweak, and build upon the work non-commercially, as long as appropriate credit is given and the new creations are licensed under the identical terms.
}

For reprints contact: WKHLRPMedknow_reprints@wolterskluwer.com
She underwent left oophorectomy for the same at an outside institution. Grossly, the left ovarian mass measured $15 \mathrm{~cm} \times 10 \mathrm{~cm} \times 7 \mathrm{~cm}$. The external surface of the tumor was bosselated with nodular areas. It had a solid cystic, grayish tan to brownish cut surface. The sections of the same were received in histopathology at our institution for review.

Microscopically on scanner view, predominant areas of the tumor showed a loose meshwork of spaces with a reticular pattern giving a sieve-like appearance, along with cystic spaces partially lined by pseudostratified columnar cells [Figure 1a and b]. On further magnification, many areas showed tumor tissue composed of round-to-oval-to-elongated cells with bipolar fibrillary processes forming perivascular pseudo-rosettes [Figure 1c and d]. The cells were arranged in nests and sheets too. Occasional true rosettes were also seen [Figure 2a]. The nuclei displayed salt-pepper chromatin [Figure 2b]. Sparse mitotic activity was seen. Psammoma bodies and foci of calcification were seen [Figure $2 \mathrm{c}$ and $\mathrm{d}$ ]. Necrosis or distinct vascular proliferation was not evident. Multiple sections examined did not show any teratomatous component.

How to cite this article: Patel TS, Samanta ST, Jana TT, Trivedi PP. Primary ependymoma of the ovary mimicking as yolk sac tumor: A rare case report. Indian J Med Paediatr Oncol 2020;41:773-6.

\section{Trupti S Patel, Satarupa T Samanta, Torsha T Jana, Priti P Trivedi}

Department of Oncopathology, Gujarat Cancer and Research Institute, Ahmedabad, Gujarat, India

Submitted: 09-Apr-2020 Revised: 04-Jun-2020

Accepted: 09-Jun-2020

Published: 29-Oct-2020

Address for correspondence: Dr. Satarupa T Samanta, Department of Oncopathology, Gujarat Cancer and Research Institute, Ahmedabad, Gujarat, India.

E-mail: samanta.satarupa@ gcriindia.org

Access this article online Website: www.ijmpo.org

DOI: 10.4103/ijmpo.ijmpo_146_20 Quick Response Code:

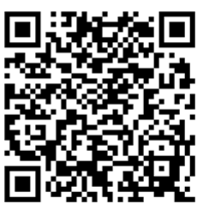




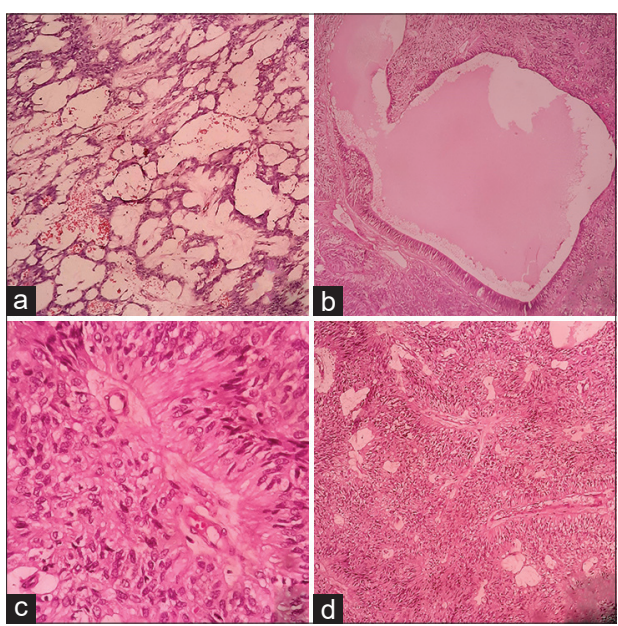

Figure 1: (a) Loose reticular pattern of arrangement of the tumor cells $(H$ and $E, \times 10)$. (b) Cystic space partially lined by pseudostratified columnar epithelium ( $\mathrm{H}$ and $\mathrm{E}, \times 10)$. (c) Tumor cell processes converging upon vessel wall in a pseudo-rosette $(H$ and $E, \times 40)$. (d) Tumor cells forming distinct perivascular pseudo-rosettes $(H$ and $E, \times 10)$

Provisional diagnosis of primary glial and neuronal tumor of the ovary was made. Serum markers CA-125, CA 19-9, CEA, AFP, and $\beta$-HCG were within normal limits. In view of extensive residual disease on follow-up abdominal CT scan and histopathological diagnosis, cytoreductive completion surgery ( total hysterectomy with right oophorectomy, bilateral salpingectomy, and staging laparotomy) was performed after obtaining informed consent from the patient.

Multiple tumor implants of similar morphology were identified over the outer surface of the posterior cervical wall, pouch of Douglas, tissue over sigmoid colon, anterior wall of rectum, omentum, and mesentery. Right ovary and bilateral fallopian tubes were unremarkable. Section from the uterus showed products of conception comprising decidual tissue, trophoblasts, and occasional villi [Figure 2e]

On immunohistochemistry (IHC) [Figure 3], the tumor cells were strongly positive for glial fibrillary acidic protein (GFAP), S100, estrogen receptor (ER), and progesterone receptor (PR), with focal strong positivity for epithelial membrane antigen (EMA) and AE1/AE3. The MIB1 index was $10 \%-15 \%$. The final diagnosis of primary neuroectodermal tumor (PNET) of the ovary - ependymoma was made. Negativity for AFP and OCT 3/4 ruled out yolk sac tumor and other germ cell tumors. Inhibin negativity ruled out sex-cord stromal tumors; negativity for PAX8 and WT1 ruled out epithelial ovarian tumors.

Subsequently, the patient was alive on hormonal therapy (anastrozole $[1 \mathrm{mg}]$ ) at 8 months postoperatively.

\section{Discussion}

PNETs of the ovary are divided into three groups: (1) differentiated group which includes ependymoma,

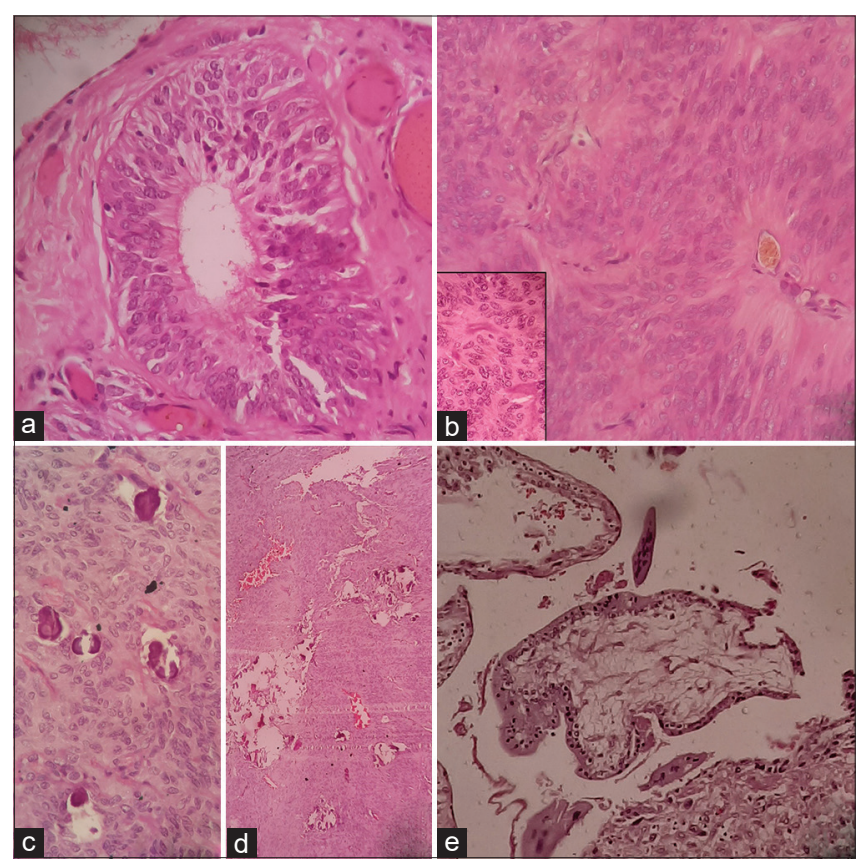

Figure 2: (a) True ependymal rosette (H and E, ×40). (b) Tumor cells with fibrillary processes. Inset: "Salt-pepper" nuclear chromatin of the tumor cells ( $H$ and $E, \times 40$ ). (c) Psammoma bodies ( $H$ and $E, \times 40)$. (d) Foci of calcification $(H$ and $E, \times 10)$. (e) Polar chorionic villi with trophoblastic cells ( $H$ and $E, \times 10)$

astrocytoma, and oligodendroglioma; (2) primitive tumors which include neuroectodermal tumors (PNETs), neuroblastoma, ependymoblastoma, medulloblastoma, and medulloepithelioma; (3) anaplastic group which includes glioblastoma multiforme. ${ }^{[4]}$

The differentiated neuroectodermal type tumor - ependymoma of the ovary occurs in a wide age range (6-69 years) but mostly occurs in young age with unilateral presentation and without extra-ovarian disease. ${ }^{[4]}$ Our patient was also a young female with unilateral presentation, however presented with extra-ovarian disease (FIGO stage IIB). Presence of extra-ovarian metastases was also documented in other cases of ovarian ependymomas in the literature. ${ }^{[1,5,6]}$ Contrary to CNS ependymomas which rarely metastasize outside the CNS, the rate of metastasis is higher in extra CNS ependymomas, due to the absence of blood-brain barrier, making it easier for the tumor cells to enter the bloodstream and lymphatic system. ${ }^{[7]}$

The histogenesis of primary ovarian ependymomas is obscure. Unlike the other neuroectodermal type tumors of the ovary, primary ovarian ependymomas are rarely associated with teratoma. ${ }^{[8]}$ Liang et al. in their study from 1988 to 2015 found 11 cases of primary glial/neuronal tumors of the ovary/peritoneum, of which four cases of ependymal tumors were not associated with another ovarian neoplasm. ${ }^{[9]}$ Multiple sections studied from our case also did not reveal any other ovarian neoplasm. Rarely, teratomas of the ovary may show large areas with 


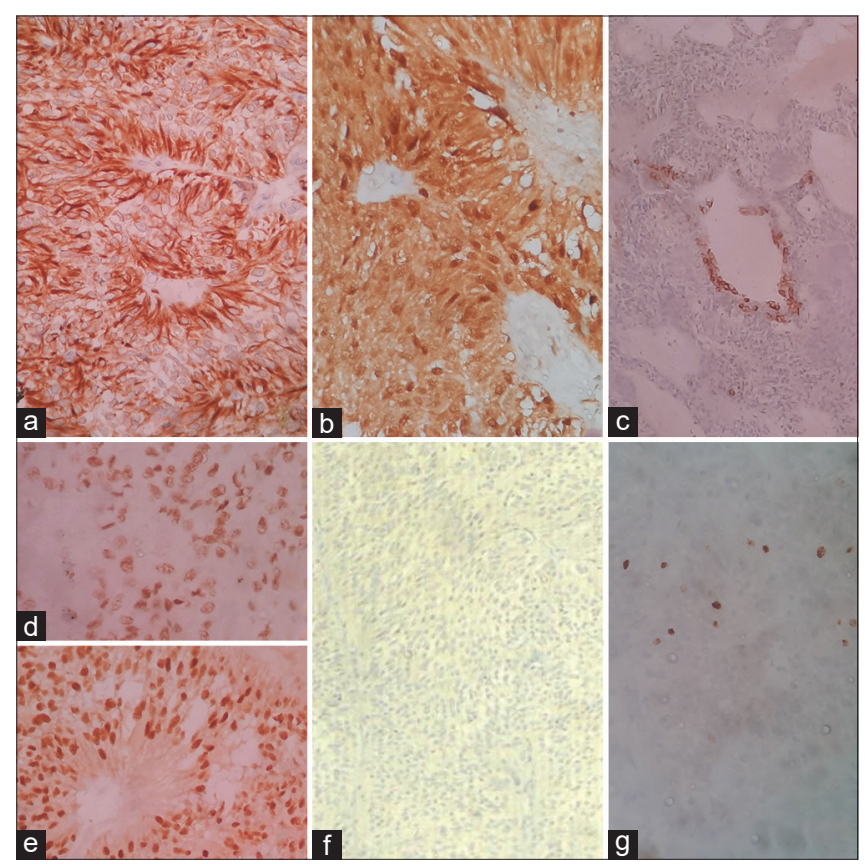

Figure 3: Tumor cells showing strong positivity for (a) glial fibrillary acidic protein, (b) S100; focal strong positivity for (c) AE1; nuclear positivity for (d) estrogen receptor and (e) progesterone receptor; negativity for (f) AFP, and (g) MIB1 was 10\%-15\%

neural differentiation composed of several neuroectodermal cell types such as astrocytes, oligodendroglial cells, and ganglion cells, resembling hamartomas. Contrarily, ovarian neuroectodermal tumors arising de novo show cellular neural differentiation comprising one or few cell types, resembling neoplasms of the nervous system, as in our case. ${ }^{[8]}$ Moreover, Stolnicu et al. found in their study that two cases of ovarian ependymomas expressed ER, PR, and cytokeratin markers, whereas the ependymoma seen with a teratoma component was negative for these markers hinting toward their derivation from distinct precursors. ${ }^{[10]}$ Our case did not show any evidence of a teratoma component and showed positivity for ER, PR, EMA, and AE1/AE3.

Ovarian neuroectodermal tumors may mimic a number of primary and metastatic ovarian tumors. Because of the young age of our patient and predominant areas demonstrating sieve-like reticular pattern, yolk sac tumor had to be ruled out. Absence of hyperchromasia, irregularly shaped and sized nuclei with nucleoli, scarcity of mitotic figures, negativity for AFP on IHC, and unelevated serum AFP levels ruled out yolk sac tumor in our case.

Sieve-like architectural pattern may also mimic ovarian tumor of probable Wolffian origin. ${ }^{[8]}$ Presence of papillae occasionally associated with psammoma bodies can be mistaken for serous carcinomas. ${ }^{[2]}$ Ependymal tubules may mimic the glands of endometrioid carcinoma. The central lumen rosettes in ependymomas may mimic CallExner bodies of the granulosa cell tumors. ${ }^{[8]}$ Ribbons of cells or tubules in an ependymoma may mimic typical or retiform variant of Sertoli-Leydig cell tumor ${ }^{[8]}$ In our case, characteristic features of ependymoma, such as rosettes, fibrillary cytoplasm, gliovascular arrangement, along with strong positivity for GFAP and S100, along with negativity for PAX 8, WT1, and inhibin, helped to arrive at the final diagnosis.

Compared to their CNS counterparts, primary ovarian ependymomas are more likely to express various cytokeratin, ER, and PR, on IHC. ${ }^{[1]}$ Positivity for both ER and PR enables treatment with hormonal therapies such as aromatase inhibitors. ${ }^{[11]}$ Our patient received hormonal therapy and has disease-free survival at 8 months postoperatively.

We had the incidental finding of intrauterine products of conception comprising decidual tissue, trophoblasts, and an occasional villi, along with primary ovarian ependymoma. A rare similar case was found in the literature, where a 25-year-old female presented at term pregnancy with bilateral ovarian ependymomas. ${ }^{[6]}$

With respect to treatment and prognosis, ependymomas in the CNS can be difficult to totally extirpate, considering their proximity to important CNS structures. Contrarily, ependymomas in the ovary can be often totally resected through surgery. Hence, patients with ependymomas in the ovary tend to have better recurrence-free and overall survival than their CNS counterparts. ${ }^{[9]}$ In our case, the patient successfully underwent complete debulking followed by hormonal therapy.

In summary, ovarian ependymomas have much better prognosis than CNS ependymomas ${ }^{[9]}$ Hence, recognition of these rare tumors by the pathologist affects therapeutic and prognostic outcomes for the patient.

\section{Declaration of patient consent}

The authors certify that they have obtained all appropriate patient consent forms. In the form the patient(s) has/have given his/her/their consent for his/her/their images and other clinical information to be reported in the journal. The patients understand that their names and initials will not be published and due efforts will be made to conceal their identity, but anonymity cannot be guaranteed.

\section{Financial support and sponsorship}

Nil.

\section{Conflicts of interest}

There are no conflicts of interest.

\section{References}

1. Idowu MO, Rosenblum MK, Wei X, Edgar MA, Soslow RA. Ependymomas of the central nervous system and adult extra-axial ependymomas are morphologically and immunohistochemically distinct-A comparative study with assessment of ovarian carcinomas for expression of glial fibrillary acidic protein. Am J Surg Pathol 2008;32:710-18.

2. Bell DA, Woodruff JM, Scully RE. Ependymoma of the broad 
ligament. A report of two cases. Am J Surg Pathol 1984;8:203-9.

3. Doglioni C, Bontempini L, Iuzzolino P, Furlan G, Rosai J. Ependymoma of the mediastinum. Arch Pathol Lab Med 1988;112:194-6.

4. Prat J, Cao D, Carinelli SG, Nogales FF, Vang R, Zaloudek CJ. Monodermal teratomas and somatic type tumours arising from a dermoid cyst. In: Kurman RJ, Carcangiu ML, Herrington CS, Young RH, editors. WHO Classification of Tumours of Female Reproductive Organs. $4^{\text {th }}$ ed. Lyon: IARC; 2014. p. 64-5.

5. Hirahara F, Yamanaka M, Miyagia E, Nakazawa T, Gorai I, Minaguchi $\mathrm{H}$, et al. Pure ovarian ependymoma: Report of a case treated with surgery, chemotherapy, irradiation and hyperthermotherapy. Eur J Obstet Gynecol Reprod Biol 1997;75:221-3.

6. Carr KA, Roberts JA, Frank TS. Progesterone receptors in bilateral ovarian ependymoma presenting in pregnancy. Hum Pathol 1992;23:962-5.
7. Yust Katz S, Cachia D, Kamiya-Matsuoka C, Olar A, Theeler B, Penas Prado $\mathrm{M}$, et al. Ependymomas arising outside of the central nervous system: A case series and literature review. J Clin Neurosci 2018;47:202-7.

8. Kleinman GM, Young RH, Scully RE. Primary neuroectodermal tumors of the ovary. A report of 25 cases. Am J Surg Pathol 1993;17:764-78.

9. Liang L, Olar A, Niu N, Jiang Y, Cheng W, Bian XW, et al. Primary glial and neuronal tumors of the ovary or peritoneum: A clinicopathologic study of 11 cases. Am J Surg Pathol 2016;40:847-56.

10. Stolnicu S, Furtado A, Sanches A, Nicolae A, Preda O, Hincu M, et al. Ovarian ependymomas of extra-axial type or central immunophenotypes. Human Pathol 2011;42:403-8.

11. Gorski JW, Taylor JS, Zhang J, Liu J, Jazaeri AA. Hormonal based treatment of ovarian anaplastic ependymoma with anastrozole. Gynecol Oncol Rep 2017;20:93-6. 